\section{Pulmonary artery occlusion by tuberculous mediastinal lymphadenopathy}

\author{
W M Drake, S L Elkin, A Al-kutoubi, \\ D M Mitchell, R J Shaw
}

\begin{abstract}
Two cases of tuberculous mediastinal lymphadenopathy are presented in which the clinical features mimicked those of pulmonary embolism, causing striking abnormalities on radionuclide scintigraphy. It is concluded that tuberculous mediastinal lymphadenopathy should be included in the differential diagnosis of reduced lung perfusion due to proximal pulmonary artery occlusion.

(Thorax 1997;52:301-302)
\end{abstract}

Keywords: granulomatous lymphadenopathy, pulmonary artery occlusion.

Granulomatous mediastinal lymphadenopathy usually presents with insidious onset of exertional dyspnoea, cough, haemoptysis, night sweats, and weight loss. Other less common presentations include superior vena caval obstruction, recurrent laryngeal nerve palsy, stridor, and dysphagia. ${ }^{1}$ We report two cases of tuberculous mediastinal lymphadenopathy in which the clinical features mimicked those of pulmonary embolism, causing striking abnormalities on radionuclide scintigraphy. This report suggests that tuberculous mediastinal lymphadenopathy should be included in the differential diagnosis of reduced lung perfusion

due to proximal pulmonary artery occlusion.

Respiratory Medicine

W M Drake

$S$ L Elkin

D M Mitchell

R J Shaw

Department of

Radiology

A Al-kutoubi

St Mary's Hospital,

Praed Street,

London W2 1NY, UK

Department of

Respiratory Medicine, Imperial College

School of Medicine at St Mary's, London

W2, UK

R J Shaw

Correspondence to:

Dr R J Shaw.

Received 21 December 1995 Returned to authors

26 March 1996

Revised version received

3 May 1996

Accepted for publication

23 May 1996

\section{Case 1}

A 36 year old Asian woman presented with a four week history of exertional dyspnoea and pleuritic chest pain. She reported no productive cough, haemoptysis, fevers, or weight loss. Examination revealed a tachycardia of $110 / \mathrm{min}$ and a respiratory rate of $25 / \mathrm{min}$, but was otherwise unremarkable. Plain chest radiography showed reduced vascular markings throughout the right lung. Arterial gases revealed hypoxia $\left(\mathrm{PaO}_{2} 9.6 \mathrm{kPa}\right)$ with a normal $\mathrm{PaCO}_{2}$ and $\mathrm{pH}$. A ventilation/perfusion (V/Q) scan showed reduced perfusion to the right lung but with normal ventilation (fig 1). Pulmonary angiography was performed; no filling defect was seen but the right main pulmonary artery was stenosed (fig 2). Spiral computed tomographic (CT) scanning showed a soft tissue mass below the aortic arch causing narrowing of the right pulmonary artery (fig 3 ). The artery was reconstructed using a polytetrafluoroethylene
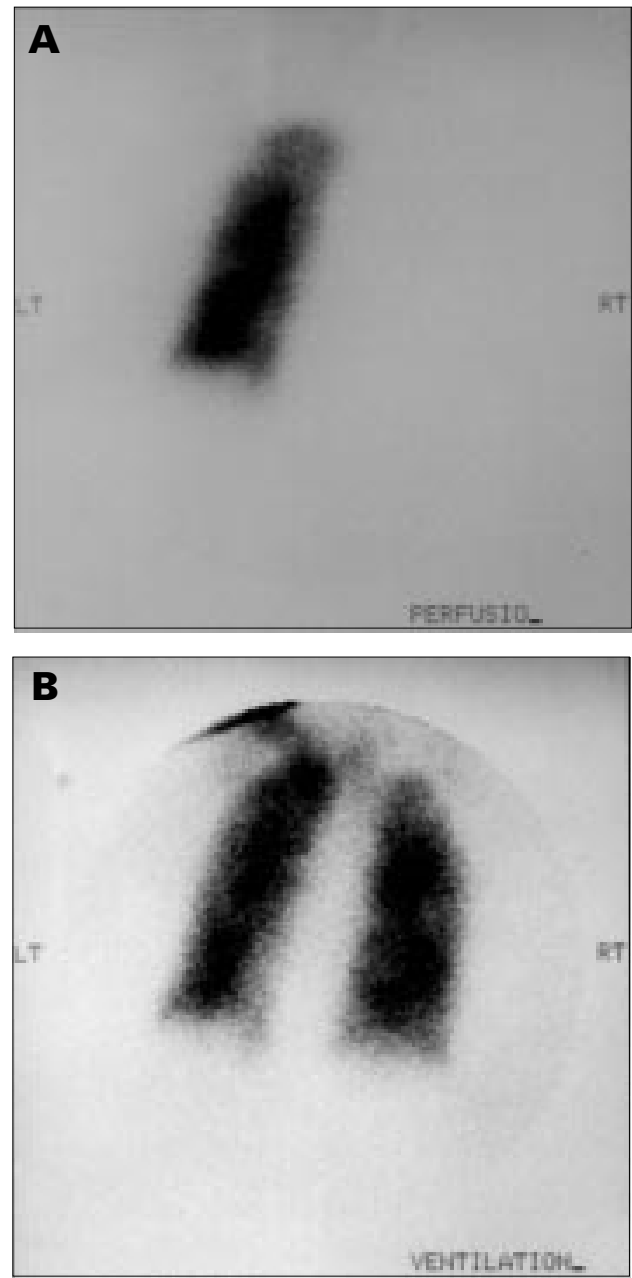

Figure 1 Ventilation/perfusion scan in case 1 (posterior view) showing $(A)$ almost total absence of perfusion to the right lung but with normal ventilation (B).

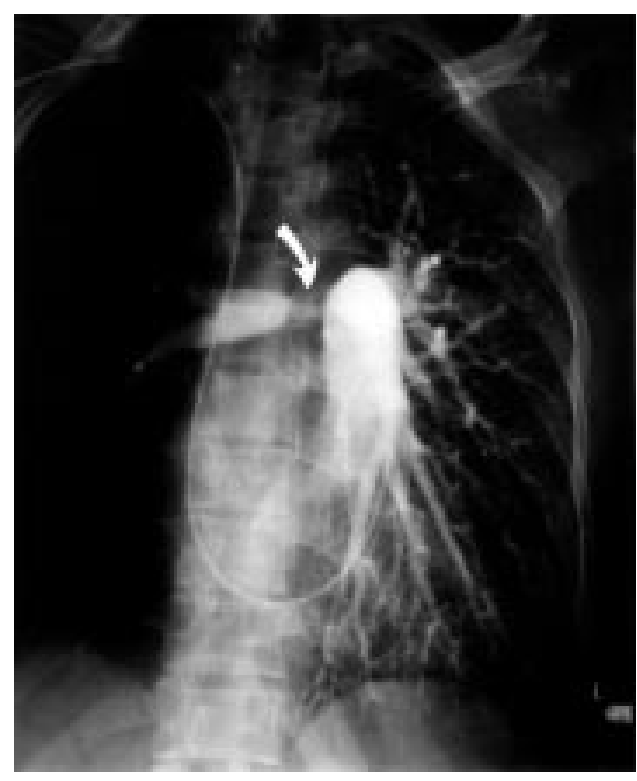

Figure 2 Pulmonary angiogram in case 1 (anterior view) showing stenosis of the right main pulmonary artery. 


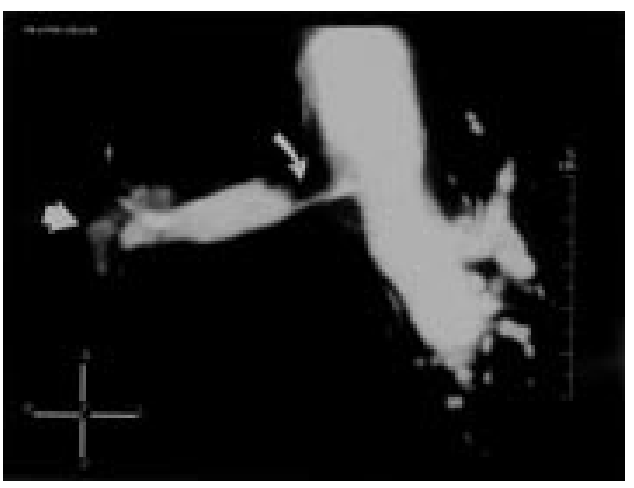

Figure 3 Figure 3 Reconstructed spiral CT scan of the pulmonary arteries in case 1 showing stenosis of the right main pulmonary artery (curved arrow). Abnormal tissue is also seen at the right hilum (arrowhead) representing involved glands.

patch graft. Histological examination of the mass revealed caseating granulomas containing acid-alcohol fast bacilli (AAFB) extending into and involving the wall of the artery. Antituberculous chemotherapy was commenced and a subsequent $\mathrm{V} / \mathrm{Q}$ scan showed restoration of perfusion to the right lung.

\section{Case 2}

A 30 year old Ethiopian woman presented with a four week history of frank haemoptysis and worsening exertional dyspnoea. She reported no fevers, night sweats, or chest pain. On examination she was well perfused with a pulse rate of $90 / \mathrm{min}$ and a blood pressure of $110 /$ $70 \mathrm{~mm} \mathrm{Hg}$. The pulmonary component of the second heart sound was accentuated, but the remainder of the examination was non-contributory. Arterial blood gas tensions and ECG findings were normal. Plain chest radiography revealed right hilar enlargement. A V/Q scan showed reduced perfusion to the whole of the right lung with normal ventilation bilaterally. Pulmonary angiography was performed; no filling defect was seen but extrinsic obstruction at the level of the right pulmonary artery was noted. A CT scan showed a soft tissue mass surrounding the right hilum, extending posteriorly into the subcarinal space and around the right main bronchus. Histological ex- amination of tissue obtained under CT guidance was non-diagnostic and no AAFB were cultured. A Heaf test was strongly positive and antituberculous chemotherapy was commenced. After eight weeks of therapy haemoptysis ceased and a repeat lung perfusion scan showed that perfusion to the right lung had significantly increased.

\section{Discussion}

Sudden occlusion of $50 \%$ or more of the pulmonary vascular tree may be associated with dramatic haemodynamic collapse characterised by tachycardia, hypotension, oliguria, and shock. In such cases pulmonary angiography or spiral CT scanning may demonstrate a filling defect in one or more proximal pulmonary arteries. In both of our cases it was the discrepancy between the clinical condition of the patient and the dramatic unilaterally abnormal appearance of the perfusion scan that prompted further investigation with CT scanning and pulmonary angiography. In each case mediastinal lymphadenopathy was demonstrated on the CT scan and pulmonary angiography showed narrowing of a proximal pulmonary artery. Clinical improvement with restoration of normal lung perfusion was demonstrated following antituberculous chemotherapy in case 2 and after surgery and antituberculous chemotherapy in case 1 .

Causes of reduced perfusion to an entire lung not due to pulmonary embolism include bronchial carcinoma, large ventilated bullae, pulmonary vasculitis, McLeod's syndrome, bronchogenic cyst, and fibrosing mediastinitis..$^{23}$ This report suggests that tuberculous mediastinal lymphadenopathy should be added to the differential diagnosis of patients who present with a clinical picture of pulmonary embolism in whom the appearances of a lung perfusion scan suggest proximal pulmonary artery occlusion.

1 Goldstraw P. Mediastinal masses. In: Brewis RAL, Lovin B, Geddes DM, Gibson GJ, eds. Respiratory medicine. London: WB Saunders, 1995:1595-7.

2 Watts WJ, Rotman HH, Patten GA. Pulmonary artery compression by a bronchogenic cyst simulating congenital pulpression by a bronchogenic cyst simulating congenital

3 Mallin WH, Silberstein EB, Shipley RT, Vu DN, Alspaugh JP, Maulton JS. Fibrosing mediastinitis causing nonvisualization of one lung on pulmonary scintigraphy. Clin Nucl Med 1993;18:594-6. 